\title{
Differentially expressed IncRNAs, miRNAs and mRNAs with associated ceRNA networks in a mouse model of myocardial ischemia/reperfusion injury
}

\author{
RUI ZHANG ${ }^{1-4}$, JIALI WANG $^{1-4}$, BAOSHAN LIU $^{1-4}$, WENJUN WANG $^{1-4}$, XINHUI FAN $^{1-4}$, \\ BOYUAN ZHENG $^{1-4}$, QIUHUAN YUAN ${ }^{1-4}$, MENGYANG XUE $^{1-4}$, \\ FENG $\mathrm{XU}^{1-4}$, PING GUO ${ }^{1-5}$ and YUGUO $\mathrm{CHEN}^{1-4}$ \\ ${ }^{1}$ Department of Emergency Medicine, ${ }^{2}$ Shandong Provincial Clinical Research Center for Emergency \\ and Critical Care Medicine, Institute of Emergency and Critical Care Medicine of Shandong University, \\ ${ }^{3}$ Key Laboratory of Emergency and Critical Care Medicine of Shandong Province, \\ Key Laboratory of Cardiopulmonary-Cerebral Resuscitation Research of Shandong Province, \\ Shandong Provincial Engineering Laboratory for Emergency and Critical Care Medicine, \\ ${ }^{4}$ Key Laboratory of Cardiovascular Remodeling and Function Research, Chinese Ministry of Education, \\ Chinese Ministry of Health and Chinese Academy of Medical Sciences; \\ The State and Shandong Province Joint Key Laboratory of Translational Cardiovascular Medicine, \\ Qilu Hospital, Shandong University; ${ }^{5}$ Medical Insurance Office, Qilu Hospital, \\ Shandong University, Jinan, Shandong 250012, P.R. China
}

Received November 15, 2019; Accepted June 5, 2020

DOI: $10.3892 / \mathrm{mmr} .2020 .11300$

\begin{abstract}
Non-coding RNAs, including long non-coding RNAs (lncRNAs) and microRNAs (miRNAs/miRs), have significant regulatory effects on a number of biological processes in myocardial ischemia/reperfusion (I/R) injury, including cell differentiation, proliferation and apoptosis. In the present study, the expression levels of lncRNAs, miRNAs and mRNAs were evaluated in a mouse model of myocardial I/R injury. The potential functions of these differentially expressed genes were then analyzed via Gene Ontology and pathway analyses. Additionally, the interactions between IncRNA-miRNA-mRNA were predicted by constructing a competing endogenous RNA regulatory network. It was found that 14,366 lncRNAs, 151 miRNAs and 9,377 mRNAs were differentially expressed in mice hearts after I/R compared with the Sham group (fold change $>2$; $\mathrm{P}<0.05$ ). The results indicated that these differentially expressed genes were involved in multiple molecular functions, including 'guanosine
\end{abstract}

Correspondence to: Professor Yuguo Chen or Professor Ping Guo, Department of Emergency Medicine, Qilu Hospital, Shandong University, 107 Wenhua Xi Road, Jinan, Shandong 250012, P.R. China E-mail: chen919085@sdu.edu.cn

E-mail: 13953100732@163.com

Key words: long non-coding RNAs, microRNAs, mRNAs, competing endogenous RNA networks, myocardial ischemia/ reperfusion injury diphosphate binding', 'RNA polymerase II carboxy-terminal domain kinase activity', 'TATA-binding protein-class protein binding', 'nicotinamide adenine dinucleotide binding' and 'protein phosphatase type $2 \mathrm{~A}$ regulator activity'. The interactions between IncRNA-miRNA-mRNA, including five lncRNAs, 38 miRNAs and 196 mRNAs, were predicted, specifically Gm12040-mmu-miR-125a-5p-decapping mRNA 1B, Rpl711-ps1-mmu-miR-124-3p-G protein-coupled receptor 146, Gm11407-mmu-miR-190a-5p-homeobox and leucine zipper encoding (HOMEZ), 1600029O15Rik-mmu-miR-132 -3p-HOMEZ and AK155692-mmu-miR-1224-3p-activating transcription factor $6 \beta$. Collectively, these findings provided novel insights for future research on lncRNAs, miRNAs and mRNAs in myocardial I/R injury.

\section{Introduction}

Acute myocardial infarction is the leading cause of morbidity and mortality worldwide and $>7$ million new patients have been diagnosed with acute myocardial infarction worldwide annually $(1,2)$. The primary therapeutic strategy for acute myocardial ischemic injury is prompt and effective reperfusion, including fibrinolytic therapy and primary percutaneous coronary intervention; however, reperfusion itself further induces myocardial injury (3). Moreover, the underlying mechanisms responsible for myocardial ischemia/reperfusion (I/R) injury are complicated and are yet to be completely elucidated; therefore, myocardial I/R injury remains an ongoing unmet clinical issue.

Previous studies have reported that non-coding RNAs serve an important role in regulating pathophysiological 
processes of myocardial I/R injury, including cell differentiation, proliferation, apoptosis, necrosis and autophagy (4-7). Non-coding RNAs are functional RNAs that are transcribed from non-coding DNAs and have important regulatory functions, although they lack the potential of protein coding (8). Among non-coding RNAs, both microRNAs (miRNAs/miRs) and long non-coding RNAs (lncRNAs) have gained increased attention for their roles in myocardial I/R injury (9). miRNAs are 20-25 nucleotides in length and can inhibit the translation of mRNAs or induce degradation of post-transcriptional RNAs (10). It has been revealed that a number of miRNAs, such as hsa-miR-124-3 and hsa-miR-9-1, are involved in myocardial I/R injury (11). Furthermore, lncRNAs, $\geq 200$ nucleotides in length, can regulate miRNA functions by acting as endogenous sponges; and miRNAs have also been shown to bind and regulate lncRNAs stability $(12,13)$. For example, the mitochondrial dynamic-related lncRNA has been reported to act as an endogenous sponge by binding to miR-361, downregulating its expression and inhibiting mitochondrial fission and apoptosis in cardiomyocytes $(14,15)$. Despite these findings, the comprehensive expression profiles of lncRNAs, miRNAs and mRNAs, as well as their individual potential functions and the regulatory networks among them, are not well characterized in myocardial I/R injury.

In the present study, the expression profiles of lncRNAs, miRNAs and mRNAs in mice hearts after myocardial I/R were investigated using microarray analysis. The potential functions of these differentially expressed genes were analyzed via bioinformatics, including Gene Ontology (GO) and pathway analyses. Moreover, lncRNA-miRNA-mRNA regulatory networks were constructed using competing endogenous RNA (ceRNA) analysis. The results provided a series of novel areas for future research on lncRNAs, miRNAs, mRNAs and their interactions in the process of myocardial I/R injury, which is important to further understand the underlying mechanisms and potential therapies for myocardial I/R injury.

\section{Materials and methods}

Animals. A total of 24 male C57BL/6J mice (age, 8 weeks, 20-25g) were purchased from the Department of Experimental Animals of Shandong University (Jinan, China). All animal procedures were in accordance with the US National Research Council Committee Guidelines (16) and were approved by the Animal Use and Care Committee of Shandong University. All mice were housed in a temperature-controlled room under a 12-h light-dark circadian cycle with temperature of $21.0 \pm 1.0^{\circ} \mathrm{C}$, humidity of $55.0 \pm 5.0 \%$ and had free access to standard chow and water.

The mice were randomly divided into sham-operation (Sham) group $(n=12)$ and I/R group $(n=12)$. Among them, four mice in Sham group and four mice in the I/R group were used for microarray analysis. The other eight mice in each group were used to assess the success of the I/R model and the results of microarray analysis.

Mouse model of myocardial I/R injury. The myocardial $\mathrm{I} / \mathrm{R}$ model was performed in mice as previously described (17). Mice were anesthetized with $1.5 \%$ isoflurane mixed with
$100 \%$ oxygen. Following the skin incision, mice hearts were exposed via a left thoracotomy in the third or fourth intercostal space. Ischemia was performed by ligating the left anterior descending artery with an 8-0 silk ligature at $1.5-2.0 \mathrm{~mm}$ below the tip of the left auricle. Mice in the Sham group underwent the same procedure without occlusion of the left anterior descending artery. After 45 min of occlusion, the suture was untied for reperfusion, and the chest cavity and skin incision were closed. After 90 min of reperfusion, mice were sacrificed by cervical dislocation and the heart was quickly removed and cut into eight 1-2 mm thick slices from the apex to the base. The peripheral region of infarct region was selected as the area at risk (18). In addition, the two adjacent slices below the site of occlusion were collected. One slice was used for 2, 3, 5-triphenyltetrazolium chloride (TTC) staining as a reference for infarct area. Then, myocardial samples of $3 \times 3 \times 3 \mathrm{~mm}$ were collected from the area at risk from the other slice based on the reference of TTC staining. Blood samples $(\sim 1 \mathrm{ml})$ were obtained using the retrobulbar technique (19). Blood samples were centrifuged at $1000 \mathrm{xg}$ for $20 \mathrm{~min}$ at $4^{\circ} \mathrm{C}$ to collect plasma.

Detection of myocardial $I / R$ injury. In order to evaluate the success of the myocardial I/R procedure, the infarct area was determined using staining with $1 \%$ TTC (cat. no. 17779; SigmaAldrich; Merck KGaA). Fresh myocardial slices of 1-2 mm were incubated with $1 \% \mathrm{TTC}$ at $37^{\circ} \mathrm{C}$ protected from light for $30 \mathrm{~min}$ and then observed using an Olympus SZ61 stereoscopic microscope (Olympus Corporation) at x10 magnification. In addition, plasma lactate dehydrogenase (LDH) and creatine kinase-MB (CK-MB) levels were detected using ELISA kits (cat. nos. SEB864Ra and SEA479Ra, respectively; Cloud-Clone Corp.), according to the manufacturer's instructions.

RNA extraction. RNA was extracted from myocardial tissue using TRIzol ${ }^{\circledR}$ reagent (Invitrogen; Thermo Fisher Scientific, Inc.). The quantification and quality of RNA were measured using NanoDrop ND-1000 (Thermo Fisher Scientific, Inc.), and the integrity of RNA was further tested by $1 \%$ denaturing agarose gel electrophoresis. For spectrophotometry, the optical density (OD) A260/A280 ratio was 2.0 for pure RNA (ratios between 1.8 and 2.1 are acceptable) (20) and the OD A260/A230 ratio was $>1.8$.

Acquisition and analysis of microarray data. The Arraystar Mouse lncRNA array v3.0 (8x60K; Arraystar Inc.) was designed to profile lncRNAs and mRNAs in the mouse genome. A total of 35,923 lncRNAs and 24,881 mRNA were collected from authoritative data sources, including RefSeq (https://www.ncbi.nlm.nih.gov/refseq/) (21), University of California Santa Cruz Known Genes (https://genome.ucsc. edu/) (22), Ensembl (http://ensemblgenomes.org/) (22), Fantom5 Cat, Gencode and BIGTranscriptome $(23,24)$. The 7th generation miRCURY LNA ${ }^{\mathrm{TM}}$ miRNA array (Exiqon; Qiagen) was designed to profile miRNA in the mice genome, it contains 3,100 capture probes, covering all human, mouse and rat miRNAs annotated in the miRBase 19.0 (http://microrna. sanger.ac.uk/) (25), as well as all viral miRNAs related to these species. 
The Agilent Feature Extraction software (version 11.0.1.1; Agilent Technologies, Inc.) was used to analyze acquired microarray images. Quantile normalization and subsequent data processing were performed using the GeneSpring GX v12.1 software package (Agilent Technologies Inc.). After quantile normalization of the raw data, lncRNAs, miRNAs and mRNAs, of which $\geq 4 / 8$ samples had flags in Present or Marginal ('All Targets Value') which indicated good quality of data, were selected for further data analysis. Differentially expressed lncRNAs, miRNAs and mRNAs with statistical significance between the two groups were identified via $\mathrm{P}$-value filtering $(\mathrm{P}<0.05)$ and fold change filtering (fold change $>2$ ). Hierarchical clustering and combined analysis were performed using the Multiple Experiment Viewer 4.9.0 (http://mev.tm4.org/) (26).

Reverse transcription-quantitative ( $R T-q) P C R$. RT-qPCR was performed to assess the results of the microarray analysis. Total RNAs, extracted from four samples in the Sham group and four samples in the I/R group using miRcute miRNA isolation kit (cat. no. DP501; Tiangen Biotech. Co.) for miRNA and TRIzol (cat. no. DP405; Tiangen Biotech. Co.) for mRNA and lncRNA, were reverse transcribed with a High-Capacity RNA-to-cDNATM kit (cat. no. 4387406; Invitrogen; Thermo Fisher Scientific, Inc.) at $42^{\circ} \mathrm{C}$ for $60 \mathrm{~min}$ followed by $95^{\circ} \mathrm{C}$ for $3 \mathrm{~min}$. RT-qPCR was performed with the IQ-SYBR ${ }^{\circledR}$ Green Supermix in a CFX96 apparatus (Bio-Rad Laboratories, Inc.). The following thermocycling conditions were used: initial denaturation at $95^{\circ} \mathrm{C}$ for $10 \mathrm{~min}$, followed by 40 cycles at $95^{\circ} \mathrm{C}$ for $10 \mathrm{sec}$ and $60^{\circ} \mathrm{C}$ for $60 \mathrm{sec}$, and final extension at $72^{\circ} \mathrm{C}$ for $5 \mathrm{~min}$. Both $\beta$-actin and U6 small nuclear (sn)RNA were used as housekeeping genes for normalization. The expression levels of lncRNA, miRNA and mRNA were measured in terms of $\mathrm{CT}$ and then normalized to $\beta$-actin and U6 snRNA using the $2^{-\Delta \Delta \mathrm{Cq}}$ method (27). The primers used for RT-qPCR were as follows: lncRNA-AK087886 forward, 5'-ACTTACGTCTGCGACCACG-3' and reverse, 3'-GGCGG AACAAACTTCAACCT-5'; miRNA-30e-3p forward, 5'-GCC TTTCAGTCGGATGTTTACAGC-3' and reverse, 3'-GCATGT TGTCACAGCTTGTGT-5'; mRNA-Olfml1 forward, 5'-GCC GAGCACCCATCTATCAA-3' and reverse, 3'-GCCACCGG AACTGTAGACAA-5'; U6 snRNA forward, 5'-CTCGCTTCG GCAGCACA-3' and reverse, 5'-AACGCTTCACGAATTT GCGT-3'; $\beta$-actin forward 5'-CTGTCTGGCGGCACCAC CAT-3' and reverse, 3'-GCAACTAAGTCATAGTCCGC-5'.

GO and pathway analysis. The GO database (http://www. geneontology.org) was used to identify significantly overrepresented 'biological processes'. The pathway analysis was based on the Kyoto Encyclopedia of Genes and Genomes database (KEGG; http://www.genome.ad.jp/kegg/), which was performed to evaluate the potential module function.

ceRNA network analysis. IncRNAs were selected from the top ten up- and downregulated lncRNAs for ceRNA analysis with rigorous standards, including fold change $>2$, $\mathrm{P}<0.05$, raw intensity $>2,000$, exon sense overlapping and natural antisense. Then, the IncRNA-miRNA-mRNA ceRNA network was constructed based on the ceRNA hypothesis that all types of RNA transcripts, such as mRNAs, transcribed pseudogenes and lncRNAs, could communicate via a new 'language' mediated by miRNA binding sites ('microRNA response elements') (28). To construct the ceRNA network, the interaction between the miRNAs and lncRNAs was predicted using Arraystar miRNA target prediction software (29) based on TargetScan 7.2 (http://www.targetscan.org/vert_72/) and miRanda (http://www.microrna.org/microrna/home. do) (30-32). High-confidence miRNA-lncRNA pairs had a TargetScan cumulative weighted context score $<-0.3$, total context score $<-0.3$, miRanda energy score $<-10$ and structure score $>140$. Interactions between miRNAs and mRNAs were predicted using TargetScan and miRDB (http://www. mirdb.org/) (miRDB score $>70)$. Hypergeometric distribution was performed for each ceRNA pair separately $(\mathrm{P}<0.05)$ and the interactions were then combined with the microarray data to construct the ceRNA relationship. The ceRNA network was visualized using Cytoscape v2.8.3 software (https://cytoscape.org/).

Statistical analysis. The data relating to infarct size, $\mathrm{LDH}$, CK-MB and RT-qPCR were presented as the mean \pm SEM from at least three independent experiments. An unpaired Student's t-test was used to analyze the data between the two groups. $\mathrm{P}<0.05$ was considered to indicate a statistically significant difference. Statistical analysis was performed using GraphPad Prism 6 software (GraphPad Software, Inc.).

\section{Results}

Infarct size, and $\mathrm{LDH}$ and $C K-M B$ levels in myocardial $I / R$ injury. TTC staining was used to evaluate infarct size (\% of left ventricle), which was significantly higher in the myocardial I/R group compared with the Sham group (Fig. 1A; 11.7 vs. $2.3 \%$ ). It was also identified that the distal area from the site of occlusion was more severe than the proximal area after I/R injury. Moreover, the levels of plasma LDH and CK-MB were significantly increased in the myocardial I/R group compared with the Sham group (Fig. 1B and C).

Differentially expressed RNAs in myocardial $I / R$ injury. The integrity of RNAs for microarray analysis was assessed using NanoDrop ND-1000 (Table SI) and denaturing agarose gel electrophoresis (Fig. S1). Using microarray analysis, 14,366 differentially expressed lncRNAs were identified in the myocardial I/R group compared with the Sham group (fold change $>2$ and $\mathrm{P}<0.05$ ) (data not shown). Among them, 9,259 1ncRNAs (64.45\%) were upregulated and 5,107 lncRNAs $(35.55 \%)$ were downregulated. A total of 151 miRNAs were also differentially expressed; 98 (64.90\%) were upregulated and $53(35.10 \%)$ were downregulated. In addition, 9,377 mRNAs were differentially expressed; 6,472 (69.02\%) were upregulated and 2,905 (30.98\%) were downregulated. Hierarchical clustering analysis identified significant changes in the cardiac expression of lncRNAs, miRNAs and mRNAs in the myocardial I/R group compared with the Sham group (Fig. 2A-C). The top ten up- and downregulated lncRNAs, miRNAs and mRNA are presented in Tables SII-SIV, respectively.

The microarray data was assessed by randomly detecting the gene expression levels of lncRNA-AK087886, miRNA-30e-3p 
A

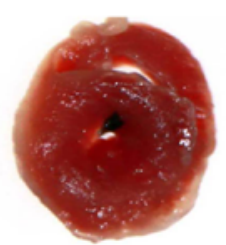

Sham

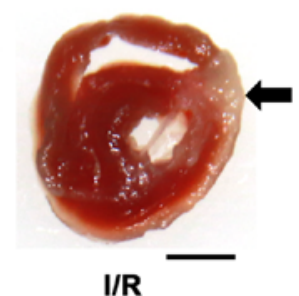

I/R

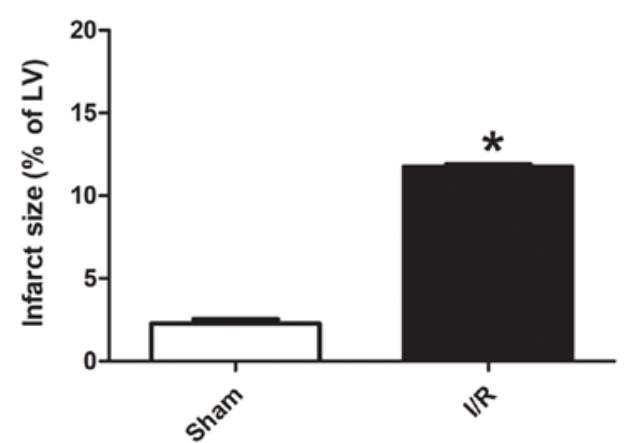

B

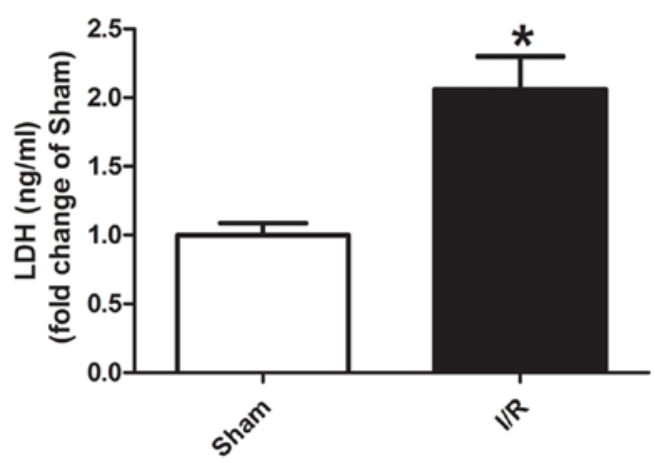

C

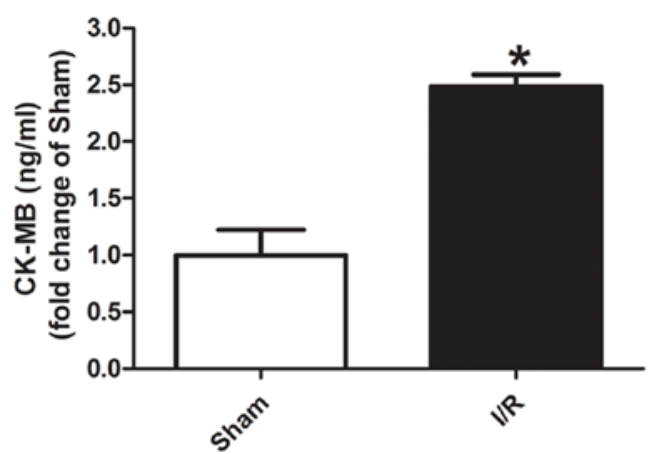

Figure 1. Myocardial I/R injury in male C57BL/6J mice. (A) Myocardial infarct area was determined using TTC staining in the Sham and I/R groups. Arrow indicates infarct area. Scale bar, $2 \mathrm{~mm}$. (B) Plasma levels of LDH in the Sham group and I/R group. (C) Plasma levels of CK-MB in the Sham group and $\mathrm{I} / \mathrm{R}$ group. Data are presented as the mean \pm SEM of $4-5$ samples in each group. ${ }^{*} \mathrm{P}<0.05$ vs. Sham group. TTC, $1 \% 2,3,5$ triphenyltetrazolium chloride; I/R, ischemia/reperfusion; LDH, lactate dehydrogenase; CK-MB, creatine kinase-MB.

A
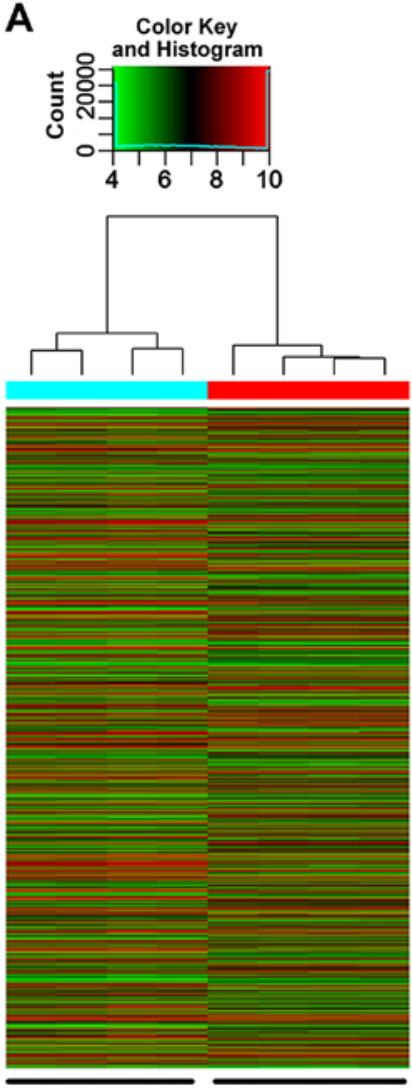

Sham

I/R
B
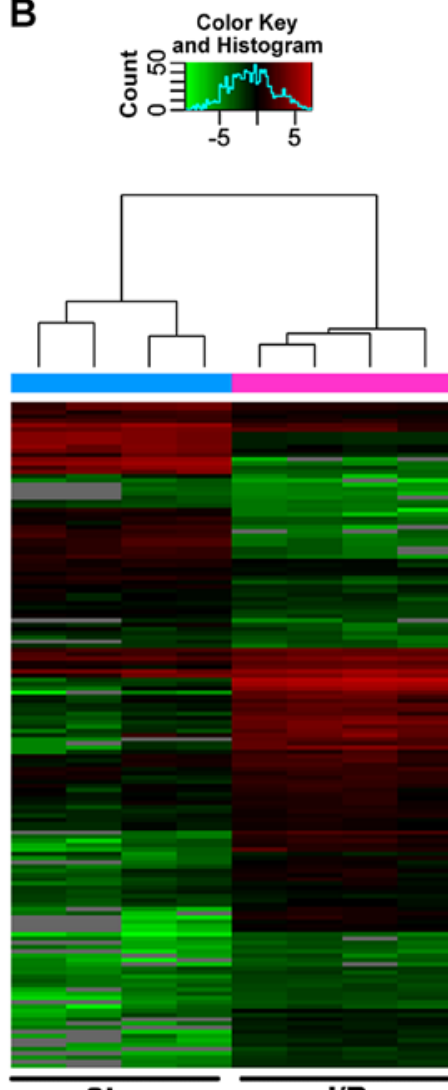

Sham
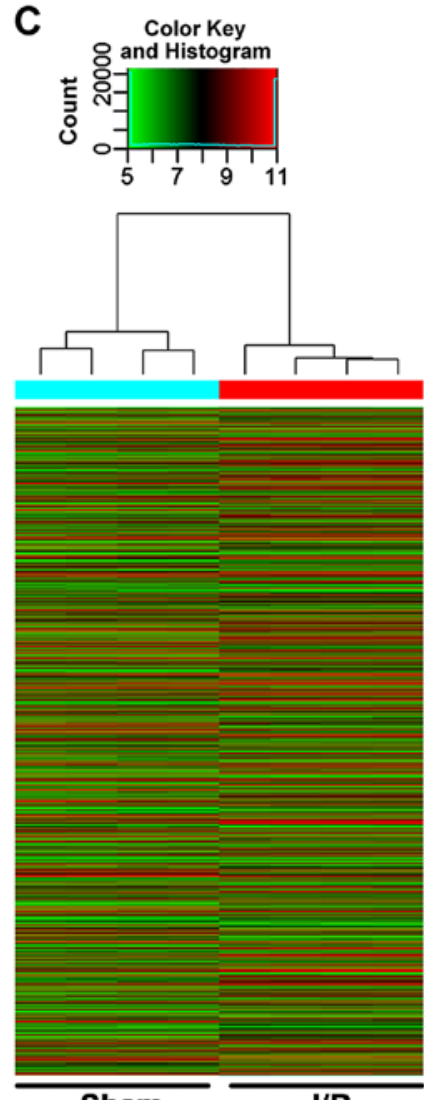

Sham

I/R

Figure 2. Heat maps constructed to determine hierarchical clustering of lncRNAs, miRNAs and mRNAs in myocardial I/R injury. Differentially expressed (A) lncRNAs, (B) miRNAs and (C) mRNAs in the Sham group and I/R group. Red represents upregulation and green represents downregulation. lncRNAs, long non-coding RNAs; miRNAs, microRNAs; I/R, ischemia/reperfusion. 
A

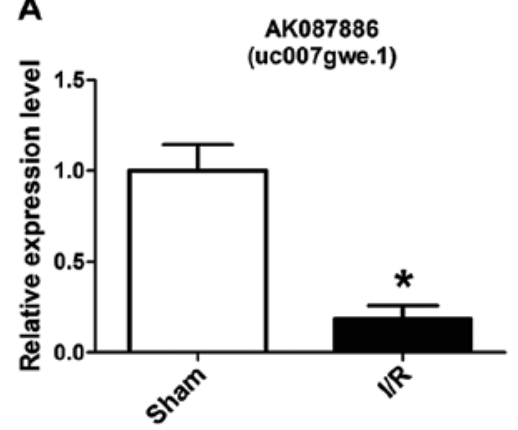

B

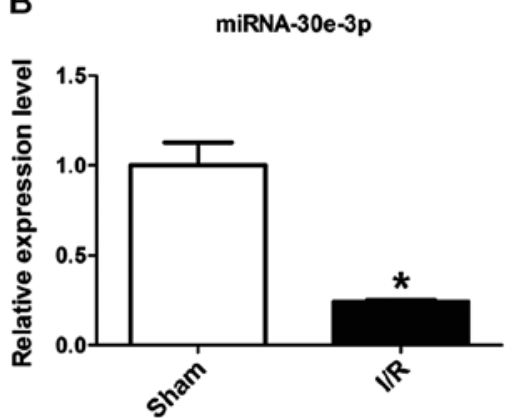

C

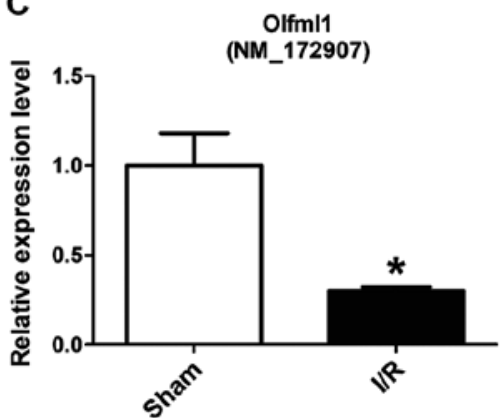

Figure 3. Validation of microarray data using reverse transcription-quantitative PCR. Relative expression levels of (A) one lncRNA, (B) one miRNA and (C) one mRNA were assessed in the Sham and I/R mice hearts. Data are presented as the mean \pm SEM of four samples in each group. * $<<0.05$ vs. Sham group. lncRNAs, long non-coding RNAs; miRNAs, microRNAs; I/R, ischemia/reperfusion; Olfml1, olfactomedin like 1.

and mRNA-Olfmll using RT-qPCR, which were in the top ten up- and downregulated lncRNAs, miRNAs and mRNAs, and the results demonstrated that the expression levels in the I/R group were significantly lower compared with those of the Sham group (Fig. 3).

GO and pathway analysis. The up- and downregulated genes were analyzed individually according to GO analysis, including 'Biological Process', 'Molecular Function' and 'Cellular Component'. The top ten molecular functions associated with up- and downregulated genes according to the enrichment score [-log10 (P-value)] are listed in Table SV. The upregulated genes were mainly involved in 'guanosine diphosphate binding', 'RNA polymerase II carboxy-terminal domain kinase activity', 'TATA-binding protein-class protein binding', 'NAD ${ }^{+}$binding' and 'protein phosphatase type $2 \mathrm{~A}$ regulator activity'. The downregulated genes were mainly involved in 'proline-rich region binding', 'sodium ion binding', 'armadillo repeat domain binding', 'alkali metal ion binding' and 'peroxisome proliferator activated receptor binding'.

Pathway analysis was performed to identify the potential module function of the differentially expressed genes in myocardial I/R injury, according to KEGG. The pathway enrichment analysis demonstrated that these upregulated genes were involved in 104 pathways and downregulated genes were involved in 29 pathways. The top ten module functions associated with up- and downregulated genes according to the enrichment score [-log10 (P-value)] are listed in Table SVI. Upregulated genes mainly participated in pathways related to 'Alzheimer's disease', 'sphingolipid signaling pathway', 'protein processing in endoplasmic reticulum', 'non-alcoholic fatty liver disease' and 'Parkinson's disease'. Downregulated genes mainly participated in pathways of 'endocytosis', 'bacterial invasion of epithelial cells', 'type II diabetes mellitus', 'oxytocin signaling pathway' and 'arrhythmogenic right ventricular cardiomyopathy'.

ceRNAs regulatory network analysis. In total, five lncRNAs, including Gm11407, AK155692, 1600029O15Rik, Rpl711-ps1 and Gm12040, were selected from the top ten up- and downregulated lncRNAs for ceRNA analysis after they were screened using rigorous standards, including fold change, P-value, raw intensity, exon sense overlapping and natural antisense. Then, the IncRNA-miRNA-mRNA ceRNA regulatory network was constructed, which included five lncRNAs, 38 miRNAs and 196 mRNAs. The IncRNA-miRNA-mRNA regulatory networks included 239 nodes and 678 edges (Fig. 4). The higher the number of nodes and edges these RNAs possessed, the increased significant effects they may have in myocardial I/R injury. Blue, red and green nodes represented lncRNAs, miRNAs and mRNAs, respectively. The edges between the nodes represented the interactions between RNAs. Furthermore, the nodes of the top ten miRNAs and mRNAs with the highest degree are presented in Table SVII. The nodes with the highest degrees in lncRNAs, miRNAs and mRNAs were Rpl711-ps1 (degree=12, indicating 12 edges or targets were directly connected to Rpl711-ps1), mmu-miR-330-3p (degree=67) and plexin A4 (Plxna4; degree=7), respectively. According to the rank scores of ceRNA5 pathways among the top 5 differentially expressed lncRNA and miRNA and mRNA (33), the following ceRNA network were chosen: Gm12040-mmu-miR-125a-5p-decapping mRNA 1B (Dcplb), Rpl711-ps1-mmu-miR-124-3p-G protein-coupled receptor 146 (Gpr146), Gm11407-mmu-miR-190a-5p-homeobox and leucine zipper encoding (HOMEZ), 1600029O15Rik-mmu-miR-132 -3p-HOMEZ and AK155692-mmu-miR-1224-3p-activating transcription factor $6 \beta$ (Atf6b).

\section{Discussion}

In the present study, the expression profiles of lncRNAs, miRNAs and mRNAs were investigated in mice hearts after I/R injury. It was found that, compared with Sham hearts, 14,366 lncRNAs, 151 miRNAs and 9,377 mRNAs were differentially expressed in the area at risk of I/R hearts. The GO and KEGG pathway analyses demonstrated the functions and pathways associated with these differentially expressed genes in myocardial I/R injury. Moreover, the interactions between IncRNA, miRNA and mRNA were constructed using ceRNA network analysis, which included five lncRNAs, 38 miRNAs and 196 mRNAs. The lncRNAs, miRNAs and mRNAs with the highest degree were lncRNA Rp1711-ps1, miRNA mmu-miR-330-3p and mRNA Plxna4. These interactions were indicated by Gm12040-mmu-miR-125a-5p-Dcp1b, Rpl711-ps1-mmu-mi R-124-3p-Gpr146, Gm11407-mmu-miR-190a-5p-HOMEZ, $1600029015 \mathrm{Rik}-\mathrm{mmu}-\mathrm{miR}-132-3 \mathrm{p}-\mathrm{HOMEZ}$ and AK155692-mmu-miR-1224-3p-Atf6b. Thus, the present study 


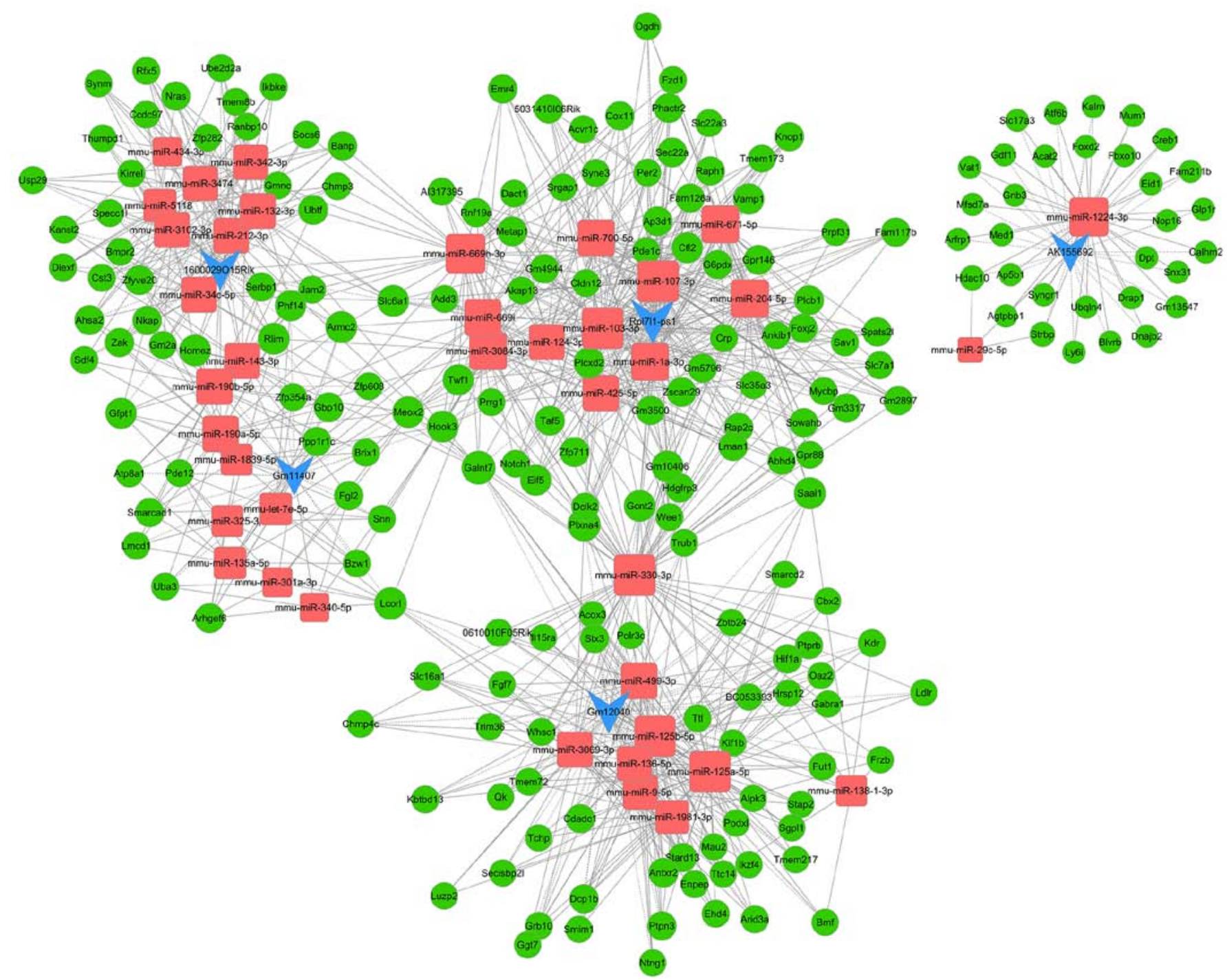

Figure 4. Competing endogenous RNA network related to myocardial ischemia/reperfusion injury. Blue, red and green nodes represented lncRNAs, miRNAs and mRNAs, respectively. lncRNAs, long non-coding RNAs; miRNAs, microRNAs.

provides a novel insight into the regulatory mechanisms and possible functions of a number of lncRNAs, miRNAs and mRNAs in myocardial I/R injury.

In the present study, the differently expressed lncRNAs, miRNAs and mRNAs after myocardial I/R injury in mice were analyzed, providing valuable information to explore the potential roles of RNAs in myocardial I/R injury in future studies. Furthermore, a number of novel lncRNAs, miRNAs and mRNAs, which had not been reported in previous studies regarding myocardial I/R injury, were screened, such as Gm11407, AK155692, 1600029O15Rik, Rpl711-ps1, Gm12040, miR-3068, miR-5624, Gm20594, Ndufb6 and Fdft1. In addition, the role of numerous RNAs screened by the present study have not been comprehensively examined in myocardial I/R injury. Among them, miR-33, as the top upregulated miRNA in the present study, has been revealed to suppress cardiac remodeling via regulation of adaptive fibrotic response in patients with heart failure (34). However, whether miR-33 has a role in inhibiting cardiac remodeling following myocardial I/R injury requires further investigation. It was also previously reported that the expression of miR-378a, which was indicated to be downregulated in the present study, is altered at an earlier stage in kidney $\mathrm{I} / \mathrm{R}$ injury compared with kidney injury molecule-1 (35). As biomarkers for the diagnosis of diseases, RNAs are important in early detection compared with protein molecules (36). However, whether miR-378a is useful for the early diagnosis of acute myocardial infarction is yet to be elucidated. Pyruvate dehydrogenase kinase 4 (Pdk4), the mRNA that was significantly increased in the present study, has been shown to promote metabolic remodeling of cardiomyocytes in late pregnancy (37), but, there is currently no evidence that Pdk4 can improve energy metabolism after myocardial I/R injury. It has been revealed that farnesyl-diphosphate farnesyltransferase 1 (Fdft1), one of the top ten downregulated mRNAs, is closely associated with lipid metabolism in obesity-related type 2 diabetes and cardiovascular disease $(38,39)$. However, the relationship between Fdft1 and lipid metabolism disorder in myocardial I/R injury has not been previously reported. Therefore, the expression profiles of differentially expressed lncRNAs, miRNAs and mRNAs identified in the present study may provide potential targets for future research regarding myocardial I/R injury. 
To the best of our knowledge, the present study was the first to predict the IncRNA-miRNA-mRNA ceRNA interactions in myocardial $I / R$ injury. It was found that Rp1711-ps1, mmu-miR-330-3p and Plxna4 were the lncRNA, miRNA and mRNA with the highest degree nodes, respectively, indicating their potential role in myocardial I/R injury. Currently, there is no report on the role of Rpl711-ps1 in any disease. Previous studies on miR-330-3p have shown that it is involved in tumors, such as lung cancer, gastric cancer and pancreatic cancer, with the exception of one study, which revealed that miR-330-3p enhanced hypertrophic response of cardiomyocytes (40-43). Combined with the present results, these findings indicate that miR-330-3p may participate in myocardial I/R injury by interacting with lncRNAs and mRNAs. Plxnax 4 has been reported to serve a role in Alzheimer's disease and modulation of tau phosphorylation, however it has not been examined in myocardial I/R injury or other cardiac diseases, and thus further investigation is required (44). The lncRNA-miRNA-mRNA ceRNA interactions were demonstrated by Gm12040-mmu-miR-125a-5p-Dcplb, Rpl711-ps1-mm u-miR-124-3p-Gpr146, Gm11407-mmu-miR-190a-5p-HOMEZ, $1600029015 \mathrm{Rik}-\mathrm{mmu}-\mathrm{miR}-132-3 \mathrm{p}-\mathrm{HOMEZ}$ and AK155692-mmu-miR-1224-3p-Atf6b in the present study. Furthermore, a previous study suggested that Gpr146 was involved in constructing the $\mathrm{C}$-peptide signaling complex in microvascular diseases of diabetes, which could attenuate cardiac contractile dysfunction in myocardial I/R injury $(45,46)$. Thus, in line with the present result, lncRNA Rpl711 may regulate Gpr146 via mmu-miR-124-3p and participate in the cardiac protective mechanisms in myocardial I/R injury. It has been shown that Atf6b can regulate the pressure overload-induced cardiac hypertrophic response, which indicates the possible role of AK155692-mmu-miR-1224-3p-Atf6b in myocardial I/R injury (47). However, to the best of our knowledge, there are currently no reports on Dcplb and HOMEZ, and additional studies are required to predict the functions of Gm12040-mmu-miR-125a-5p-Dcp1b, Gm11407-mmu-miR-190a-5p-HOMEZ or 1600029O15Rik-m mu-miR-132-3p-HOMEZ.

Previous studies have investigated the expression profiles of lncRNAs, mRNAs and miRNAs in myocardial I/R injury, the majority of which have focused on the interactions between two types of RNA. For example, Liu et al (18) identified 151 differentially expressed lncRNAs and 110 mRNAs in the infarct region after myocardial I/R injury in mice, and function analysis revealed that these differentially expressed transcripts were associated with 'immune response', 'spermine catabolic process', 'taxis', 'cytokine-cytokine receptor interaction', 'the chemokine signaling pathway' and 'nucleotide oligomerization domain-like receptor signaling pathway'. Moreover, Wu et al (48) reported that 168 lncRNAs and 126 mRNAs were differentially expressed after myocardial infarction in mice, and these differentially expressed genes were enriched in 41 signaling pathways, including 'complement and coagulation cascades', 'cytokine-cytokine receptor interaction' and 'chemokine signaling pathways'. Gao et al (49) also demonstrated that 1,197 lncRNAs and 2,066 mRNAs were upregulated, whereas 1,403 lncRNAs and 2,871 mRNAs were downregulated after ischemic heart failure in rats, which were closely related to the 'mitogen-activated protein kinase-signaling pathway', 'T cell receptor signaling pathway', 'prion diseases' and 'cell adhesion molecules'. In addition, Liu et al (50) investigated the time-course of lncRNA and mRNA expression in the peripheral blood of patients with acute ST-segment elevation myocardial infarction and percutaneous coronary intervention. These authors reported 135 RNAs that were significantly associated with myocardial $\mathrm{I} / \mathrm{R}$ injury, and that mRNA SH2 domain containing $3 \mathrm{C}$ and general transcription factor IIH subunit 4 may be the most responsive transcriptional regulators in the early-phase of myocardial I/R injury (50). The present study expanded on these findings by investigating the interactions between three types of RNA, and in line with previous studies, provided a potential research area for future studies on non-coding RNAs in myocardial I/R injury.

The present results provided information on RNAs that could be used for the diagnosis and prognosis of acute myocardial infarction. IncRNAs and miRNAs have been identified as possible biomarkers for diagnosis and prognosis of diseases, especially in cancer $(51,52)$. A previous study also revealed that IncRNA HOX transcript antisense RNA acted as a potential biomarker for the prognosis of patients with squamous cell carcinoma of the head and neck (53). Moreover, Zhong et al (54) reported that lncRNAs, including taurine upregulated 1, SPRY4 intronic transcript 1 and hepatocellular carcinoma upregulated lncRNA, may serve as moderate predictors of survival in human cancer. However, biomarkers for the diagnosis of myocardial infarction are still mainly focused on protein molecules, including plasma troponin and CK-MB (55). High-sensitive cardiac troponin I has been used to improve the sensitivity of the diagnosis of acute myocardial infarction, but at the cost of lower specificity (56). Therefore, identifying novel biomarkers for the accurate diagnosis of myocardial infarction is of great significance. Currently some miRNAs, such as miR-1, miR-499 and miR-133, have been revealed as biomarkers for the diagnosis of acute myocardial infarction (57). miR-208a, expressed specifically in cardiomyocytes, also has a high sensitivity and specificity for myocardial infarction diagnosis (58). However, additional lncRNAs and miRNAs that could be used as potential biomarkers for the diagnosis and prognosis of acute myocardial infarction are yet to be elucidated.

In conclusion, the present results demonstrated the expression profiles of differentially expressed lncRNAs, miRNAs and mRNAs, as well as predicted their functions and potential pathways in myocardial I/R injury. Furthermore, the IncRNA-miRNA-mRNA ceRNA interaction networks, including five lncRNAs, 38 miRNAs and 196 mRNAs, were predicted in myocardial I/R injury. However, a limitation of the present study was that intervention experiments of RNAs were not included, and further verifications on the potential functions of these RNAs in myocardial I/R injury were not performed.

\section{Acknowledgements}

Not applicable.

\section{Funding}

This study was supported by the National Key Research and Development Program of China (grant nos. 2017YFC0908700 
and 2017YFC0908703), National Natural Science Foundation of China (grant nos. 81772036, 81671952, 81873950, 81873953, 81570401 and 81571934), National Science and Technology Fundamental Resources Investigation Project (grant nos. 2018FY100600 and 2018FY100602), Taishan Pandeng Scholar Program of Shandong Province (grant no.tspd20181220), Taishan Young Scholar Programof Shandong Province (grant nos. tsqn20161065 and tsqn201812129), Key Research and Development Program of Shandong Province (grant no. 2018GSF118003) and the Fundamental Research Funds of Shandong University (grant no. 2018JC011).

\section{Availability of data and materials}

The datasets used and/or analyzed during the current study are available from the corresponding author on reasonable request.

\section{Authors' contributions}

YC, PG, RZ, JW and BL contributed to the conception and design. RZ, BL, WW, XF and BZ performed the experiments. RZ, BL, JW, QY, MX and FX contributed to the acquisition, analysis and interpretation of data, drafted the article or revised it critically for important intellectual content. All authors reviewed and approved the final version.

\section{Ethics approval and consent to participate}

All animal procedures were approved by the Animal Use and Care Committee of Shandong University.

\section{Patient consent for publication}

Not applicable.

\section{Competing interests}

The authors declare that they have no competing interests.

\section{References}

1. Reed GW, Rossi JE and Cannon CP: Acute myocardial infarction. Lancet 389: 197-210, 2017.

2. Virani SS, Alonso A, Benjamin EJ, Bittencourt MS, Callaway CW, Carson AP, Chamberlain AM, Chang AR, Cheng S, Delling FN, et al; American Heart Association Council on Epidemiology and Prevention Statistics Committee and Stroke Statistics Subcommittee: Heart Disease and Stroke Statistics-2020 Update: A Report From the American Heart Association. Circulation 141: el39-e596, 2020.

3. Yellon DM and Hausenloy DJ: Myocardial reperfusion injury. N Engl J Med 357: 1121-1135, 2007.

4. Huang Y: The novel regulatory role of lncRNA-miRNA-mRNA axis in cardiovascular diseases. J Cell Mol Med 22: 5768-5775, 2018.

5. Ma M, Hui J, Zhang QY, Zhu Y, He Y and Liu XJ: Long non-coding RNA nuclear-enriched abundant transcript 1 inhibition blunts myocardial ischemia reperfusion injury via autophagic flux arrest and apoptosis in streptozotocin-induced diabetic rats. Atherosclerosis 277: 113-122, 2018.

6. Zhang W, Li Y and Wang P: Long non-coding RNA-ROR aggravates myocardial ischemia/reperfusion injury. Braz J Med Biol Res 51: e6555, 2018.

7. Ren L, Chen S, Liu W, Hou P, Sun W and Yan H: Downregulation of long non-coding RNA nuclear enriched abundant transcript 1 promotes cell proliferation and inhibits cell apoptosis by targeting miR-193a in myocardial ischemia/reperfusion injury. BMC Cardiovasc Disord 19: 192, 2019.
8. Wilusz JE, Sunwoo H and Spector DL: Long noncoding RNAs: Functional surprises from the RNA world. Genes Dev 23: 1494-1504, 2009.

9. Archer K, Broskova Z, Bayoumi AS, Teoh JP, Davila A, Tang Y, Su H and Kim IM: Long Non-Coding RNAs as Master Regulators in Cardiovascular Diseases. Int J Mol Sci 16: 23651-23667, 2015.

10. Wei C, Luo T, Zou S, Zhou X, Shen W, Ji X, Li Q and Wu A: Differentially expressed lncRNAs and miRNAs with associated ceRNA networks in aged mice with postoperative cognitive dysfunction. Oncotarget 8: 55901-55914, 2017.

11. Li Y, He XN, Li C, Gong L and Liu M: Identification of Candidate Genes and MicroRNAs for Acute Myocardial Infarction by Weighted Gene Coexpression Network Analysis. BioMed Res Int 2019: 5742608, 2019.

12. Blythe AJ, Fox AH and Bond CS: The ins and outs of IncRNA structure: How, why and what comes next? Biochim Biophys Acta 1859: 46-58, 2016.

13. Li S, Chen X, Liu X, Yu Y, Pan H, Haak R, Schmidt J, Ziebolz D and Schmalz G: Complex integrated analysis of lncRNAs-miRNAs-mRNAs in oral squamous cell carcinoma. Oral Oncol 73: 1-9, 2017.

14. Li N, Ponnusamy M, Li MP, Wang K and Li PF: The Role of MicroRNA and LncRNA-MicroRNA Interactions in Regulating Ischemic Heart Disease. J Cardiovasc Pharmacol Ther 22: 105-111, 2017.

15. Wang K, Sun T, Li N, Wang Y, Wang JX, Zhou LY, Long B, Liu CY, Liu F and Li PF: MDRL IncRNA regulates the processing of miR-484 primary transcript by targeting miR-361. PLoS Genet 10: e1004467, 2014.

16. National Research Council (US) Committee for the Update of the Guide for the Care and Use of Laboratory Animals: Guide for the Care and Use of Laboratory Animals. The National Academies Collection: Reports funded by National Institutes of Health. National Academies Press, Washington, DC, 2011. https://www. ncbi.nlm.nih.gov/books/NBK54050/doi: 10.17226/12910.

17. Xue L, Yang F, Han Z, Cui S, Dai S, Xu F, Zhang C, Wang X, Pang J, Pan C, et al: ALDH2 mediates the dose-response protection of chronic ethanol against endothelial senescence through SIRT1/p53 pathway. Biochem Biophys Res Commun 504: 777-783, 2018.

18. Liu Y,Li G,Lu H,Li W,Li X,Liu H,Li X,Li T and Yu B: Expression profiling and ontology analysis of long noncoding RNAs in post-ischemic heart and their implied roles in ischemia/reperfusion injury. Gene 543: 15-21, 2014.

19. Shimizu T, Suzuki S, Sato A, Nakamura Y, Ikeda K, Saitoh S, Misaka S, Shishido T, Kubota I and Takeishi Y: Cardio-protective effects of pentraxin 3 produced from bone marrow-derived cells against ischemia/reperfusion injury. J Mol Cell Cardiol 89B: B306-:B313, 2015.

20. Imbeaud S, Graudens E, Boulanger V, Barlet X, Zaborski P, Eveno E, Mueller O, Schroeder A and Auffray C: Towards standardization of RNA quality assessment using user-independent classifiers of microcapillary electrophoresis traces. Nucleic Acids Res 33: e56, 2005.

21. O'Leary NA, Wright MW, Brister JR, Ciufo S, Haddad D, McVeigh R, Rajput B, Robbertse B, Smith-White B, Ako-Adjei D, et al: Reference sequence (RefSeq) database at NCBI: Current status, taxonomic expansion, and functional annotation. Nucleic Acids Res 44D: D733-D745, 2016.

22. Singh A, Shannon CP, Kim YW, Yang CX, Balshaw R, Cohen Freue GV, Gauvreau GM, FitzGerald JM, Boulet LP, O'Byrne PM, et al: Novel Blood-based Transcriptional Biomarker Panels Predict the Late-Phase Asthmatic Response. Am J Respir Crit Care Med 197: 450-462, 2018.

23. Haas BJ, Papanicolaou A, Yassour M, Grabherr M, Blood PD, Bowden J, Couger MB, Eccles D, Li B, Lieber M, et al: De novo transcript sequence reconstruction from RNA-seq using the Trinity platform for reference generation and analysis. Nat Protoc 8: 1494-1512, 2013.

24. Wu Q, Guo L, Jiang F, Li L, Li Z and Chen F: Analysis of the miRNA-mRNA-lncRNA networks in $\mathrm{ER}^{+}$and $\mathrm{ER}^{-}$breast cancer cell lines. J Cell Mol Med 19: 2874-2887, 2015.

25. Griffiths-Jones S, Grocock RJ, van Dongen S, Bateman A and Enright AJ: miRBase: microRNA sequences, targets and gene nomenclature. Nucleic Acids Res 34: D140-D144, 2006.

26. Guzzi PH, Tradigo G and Veltri P: Using miRNA-Analyzer for the Analysis of miRNA Data. Microarrays (Basel) 5: 5, 2016.

27. Livak KJ and Schmittgen TD: Analysis of relative gene expression data using real-time quantitative PCR and the 2(-Delta Delta C(T)) Method. Methods 25: 402-408, 2001. 
28. Salmena L, Poliseno L, Tay Y, Kats L and Pandolfi PP: A ceRNA hypothesis: The Rosetta Stone of a hidden RNA language? Cell 146: 353-358, 2011.

29. Bo H, Liu Z, Tang R, Gong G, Wang X, Zhang H, Zhu F, Zhou D, Zhu W, Tan Y, et al: Testicular biopsies microarray analysis reveals circRNAs are involved in the pathogenesis of non-obstructive azoospermia. Aging (Albany NY) 12: 2610-2625, 2020.

30. Agarwal V, Bell GW, Nam JW and Bartel DP: Predicting effective microRNA target sites in mammalian mRNAs. eLife 4 4, 2015.

31. Enright AJ, John B, Gaul U, Tuschl T, Sander C and Marks DS: MicroRNA targets in Drosophila. Genome Biol 5: R1, 2003.

32. Li JH, Liu S, Zhou H, Qu LH and Yang JH: starBase v2.0: Decoding miRNA-ceRNA, miRNA-ncRNA and protein-RNA interaction networks from large-scale CLIP-Seq data. Nucleic Acids Res 42D: D92-D97, 2014.

33. Tay Y, Kats L, Salmena L, Weiss D, Tan SM, Ala U, Karreth F, Poliseno L, Provero P, Di Cunto F, et al: Coding-independent regulation of the tumor suppressor PTEN by competing endogenous mRNAs. Cell 147: 344-357, 2011.

34. Zhang $X$ and Fernández-Hernando C: miR-33 Regulation of Adaptive Fibrotic Response in Cardiac Remodeling. Circ Res 120: 753-755, 2017.

35. Zou YF, Wen D, Zhao Q, Shen PY, Shi H, Zhao Q, Chen YX and Zhang W: Urinary MicroRNA-30c-5p and MicroRNA-192-5p as potential biomarkers of ischemia-reperfusion-induced kidney injury. Exp Biol Med (Maywood) 242: 657-667, 2017.

36. Xi X, Li T, Huang Y, Sun J, Zhu Y, Yang Y and Lu ZJ: RNA Biomarkers: Frontier of Precision Medicine for Cancer. Noncoding RNA 3: 3, 2017.

37. Liu LX, Rowe GC, Yang S, Li J, Damilano F, Chan MC, Lu W, Jang C, Wada S, Morley M, et al: PDK4 Inhibits Cardiac Pyruvate Oxidation in Late Pregnancy. Circ Res 121: 1370-1378, 2017.

38. Ding J, Reynolds LM, Zeller T, Müller C, Lohman K, Nicklas BJ, Kritchevsky SB, Huang Z, de la Fuente A, Soranzo N, et al: Alterations of a Cellular Cholesterol Metabolism Network Are a Molecular Feature of Obesity-Related Type 2 Diabetes and Cardiovascular Disease. Diabetes 64: 3464-3474, 2015.

39. Ligthart S, Vaez A, Hsu YH, Stolk R, Uitterlinden AG, Hofman A, Alizadeh BZ, Franco $\mathrm{OH}$ and Dehghan A; Inflammation Working Group of the CHARGE Consortium; PMI-WG-XCP LifeLines Cohort Study: Bivariate genome-wide association study identifies novel pleiotropic loci for lipids and inflammation. BMC Genomics 17: 443, 2016.

40. Chen T, Yang Z, Liu C, Wang L, Yang J, Chen L and Li W: Circ 0078767 suppresses non-small-cell lung cancer by protecting RASSF1A expression via sponging miR-330-3p. Cell Prolif 52: e12548, 2019

41. Wang Z, Qu H, Gong $\mathrm{W}$ and Liu A: Up-regulation and tumor-promoting role of SPHK1 were attenuated by miR-330-3p in gastric cancer. IUBMB Life 70: 1164-1176, 2018.

42. Xiong X, Shi Q, Yang X, Wang W and Tao J: LINC00052 functions as a tumor suppressor through negatively modulating miR-330-3p in pancreatic cancer. J Cell Physiol 234: 15619-15626, 2019.

43. Chen Y, Liu X, Chen L, Chen W, Zhang Y, Chen J, Wu X, Zhao Y, Wu X and Sun G: The long noncoding RNA XIST protects cardiomyocyte hypertrophy by targeting miR-330-3p. Biochem Biophys Res Commun 505: 807-815, 2018.
44. Jun G, Asai H, Zeldich E, Drapeau E, Chen C, Chung J, Park JH, Kim S, Haroutunian V, Foroud T, et al: PLXNA4 is associated with Alzheimer disease and modulates tau phosphorylation. Ann Neurol 76: 379-392, 2014

45. Yosten GL, Kolar GR, Redlinger LJ and Samson WK: Evidence for an interaction between proinsulin C-peptide and GPR146. J Endocrinol 218: B1-B8, 2013.

46. Young LH, Ikeda Y, Scalia R and Lefer AM: C-peptide exerts cardioprotective effects in myocardial ischemia-reperfusion. Am J Physiol Heart Circ Physiol 279: H1453-H1459, 2000.

47. Correll RN, Grimes KM, Prasad V, Lynch JM, Khalil H and Molkentin JD: Overlapping and differential functions of ATF6a versus ATF6 $\beta$ in the mouse heart. Sci Rep 9: 2059, 2019.

48. Wu T, Wu HD, Xu ZX, Han F, Zhang BQ, Sun J and Hu SJ: Abnormal expression of long non-coding RNAs in myocardial infarction. Heart Vessels 32: 1253-1261, 2017.

49. Gao W, Wang ZM, Zhu M, Lian XQ, Zhao H, Zhao D, Yang ZJ, $\mathrm{Lu} X$ and Wang LS: Altered long noncoding RNA expression profiles in the myocardium of rats with ischemic heart failure. J Cardiovasc Med (Hagerstown) 16: 473-479, 2015.

50. Liu $\mathrm{H}, \mathrm{Xu} \mathrm{D}$, Zhong $\mathrm{X}, \mathrm{Xu} \mathrm{D}$, Chen G, Ge J and Li $\mathrm{H}$ : LncRNA-mRNA competing endogenous RNA network depicts transcriptional regulation in ischaemia reperfusion injury. J Cell Mol Med 23: 2272-2276, 2019.

51. Fu LL, Li CJ, Xu Y, Li LY, Zhou X, Li DD, Chen SX, Wang FG, Zhang XY and Zheng LW: Role of lncRNAs as Novel Biomarkers and Therapeutic Targets in Ovarian Cancer. Crit Rev Eukaryot Gene Expr 27: 183-195, 2017.

52. White NM and Maher CA: The potential use of lncRNAs found in the 8q24 region as biomarkers for colon cancer. Ann Oncol 28: 1688-1689, 2017.

53. Troiano G, Caponio VCA, Boldrup L, Gu X, Muzio LL, Sgaramella N, Wang L and Nylander K: Expression of the long non-coding RNA HOTAIR as a prognostic factor in squamous cell carcinoma of the head and neck: A systematic review and meta-analysis. Oncotarget 8: 73029-73036, 2017.

54. Zhong Y, Chen Z, Guo S, Liao X, Xie H, Zheng Y, Cai B, Huang P, Liu Y, Zhou Q, et al: TUG1, SPRY4-IT1, and HULC as valuable prognostic biomarkers of survival in cancer: A PRISMA-compliant meta-analysis. Medicine (Baltimore) 96: e8583, 2017.

55. Kehl DW, Iqbal N, Fard A, Kipper BA, De La Parra Landa A and Maisel AS: Biomarkers in acute myocardial injury. Transl Res 159: 252-264, 2012.

56. Keller T, Zeller T, Peetz D, Tzikas S, Roth A, Czyz E, Bickel C, Baldus S, Warnholtz A, Fröhlich M, et al: Sensitive troponin I assay in early diagnosis of acute myocardial infarction. N Engl J Med 361: 868-877, 2009.

57. Wang $\mathrm{C}$ and Jing Q: Non-coding RNAs as biomarkers for acute myocardial infarction. Acta Pharmacol Sin 39: 1110-1119, 2018.

58. Wang GK, Zhu JQ, Zhang JT, Li Q, Li Y, He J, Qin YW and Jing Q: Circulating microRNA: A novel potential biomarker for early diagnosis of acute myocardial infarction in humans. Eur Heart J 31: 659-666, 2010.

This work is licensed under a Creative Commons Attribution-NonCommercial-NoDerivatives 4.0 International (CC BY-NC-ND 4.0) License. 